\title{
TOLERÂNCIA A AGENTE CURARIZANTE PROVOCADA PELA ADMINISTRAÇ.ÃO REPETIDA DA DROGA
}

\author{
antonio Carlos Zanini * \\ Octavio Slemer ** \\ José EdMILSON FERNANDES ***
}

Estruturas envolvidas na transmissão do impulso nervoso podem alterar sua função normal em estado de uso ou solicitação excessiva ${ }^{11}$ sendo tal fato facilmente evidenciado em estruturas periféricas ${ }^{2}$. Assim, já se conhece há tempo o fenômeno de hipersensibilidade à acetilcolina provocada em animais pelo bloqueio contínuo da junção neuromuscular ${ }^{3,6}$. O fenômeno oposto, isto é, a diminuição da transmissão nervosa pela presença continua de substância curarizante no sangue foi sugerido como responsável pela instalação da miastenia grave, não tendo sido entretanto comprovado ${ }^{8}$.

$\mathrm{Na}$ tentativa de evidenciar tal fenômeno, em experiência anterior foi administrado diariamente a ratos o dimet́il-eter da d-tubocurarina (DMT) durante oito semanas, sendo a força dos animais medida também diariamente por provas diversas; os resultados obtidos sugerem que doses muito pequenas, por um lado, e doses próximas da dose mínima letal, por outro lado, provocam alterações funcionais da junção neuromuscular ${ }^{14}$.

A presente pesquisa foi programada na tentativa de melhor estudar as alterações funcionais observadas, utilizando-se técnicas mais precisas de medida de força e função da junção neuromuscular.

\section{MATERIAL E MÉTODO}

Quarenta ratos Wistar albinos machos, nascidos no mesmo dia e criados em condições idênticas, pesando ao redor de $350 \mathrm{~g}$, foram divididos em quatro grupos de 10 e submetidos diariamente, durante seis semanas, ao seguinte tratamento: Grupo I - nenhum tratamento, mantidas apenas as mesmas condições de dieta e habitação; Grupo II - administração i.p. de $1 \mathrm{ml}$ de solução físiológica; Grupo III - administração i.p. de $2,8 \mathrm{mcg} / \mathrm{kg}$ de dimetil éter da d-tubocurarina (DMT); Grupo IV - administração i.p. de $14 \mathrm{mcg} / \mathrm{kg}$ de DMT. As doses de DMT foram determinadas previamente em ensaio piloto no qual foi utilizado um outro lote de animais, selecionando-se uma dose alta de $14 \mathrm{mcg} / \mathrm{kg}$, capaz de provocar bloqueio

Trabalho do Departamento de Terapêutica Clínica da Faculdade de Medicina e do Departamento de Farmacologia do Instituto de Ciências Biomédicas, Universidade de São Paulo: * Professor; ** Técnico farmacologista; *** Assistente de Terapêutica Clínica.

Trabalho realizado com auxílio da Fundação de Amparo a Pesquisa no Estado de São Paulo (Auxílio 68/002). 
intenso (grupo IV) e uma dose que não provocasse nenhum efeito objetivo de 2,8 $\mathrm{mcg} / \mathrm{kg}$ (grupo III).

Decorrida uma semana da última administração da droga, os ratos foram utilizados para montagem da preparacão neuromuscular ciático-gastrocnêmico após anestesia pelo hidrato de cloral $300 \mathrm{mg} / \mathrm{kg}$ i.p. Os estímulos foram constituidos de pulsos retangulares supra-maximais de $0,5 \mathrm{mseg}$. de duração, fornecidos por estimulador Tektronix, série 160, através de unidade de isolamento Grass. Foi a seguir medido o limiar mínimo de excitabilidade a pulsos isolados na freqüência de um a cada três segundos. A seguir, media-se o estímulo máximo, utilizando-se no decorrer da experiência pulsos supra-maximais.

A força da contração isométrica foi medida em poligrafo Grass: registrava-se inicialmente a resposta a pulsos isolados $(0,33 / \mathrm{seg})$ e, a seguir, a resposta a uma salva de 10 pulsos na freqüência de $70 / \mathrm{seg}$ e repetidos a cada três segundos. A contração tetanizante foi provocada por estímulos na freqüência de 70/pulsos/seg, mantidos durante 15 segundos, após o que a preparação era novamente estimulada por pulsos isolados $(0,33 \mathrm{pulsos} / \mathrm{seg})$, medindo-se a facilitação pós-tetânica.

O potencial de ação do músculo foi simultaneamente captado e integrado em integrador Grass e registrado pelo polígrafo.

Para maior rigor na determinação da dose curarizante, foi utilizada a técnica da "terceira parte cega", sendo todas as preparações e medidas feitas por um de nós (O.S.), mantido cego quanto ao grupo que pertencia cada animal.

O DMT foi administrado por via intravenosa em doses fixas de $0,25 \mathrm{mcg}$ repetidas a intervalos de dois minutos até que se alcançasse bloqueio significativo da transmissão neuromuscular, com redução da força em torno de $80 \%$. A dose efetiva foi considerada como sendo a soma total da posologia de DMT aplicada. Em seguida, foi administrada neostigmina até se obter ampla recuperação da preparação.

Além dos 40 animais submetidos rigorosamente à mesma rotina de experiência, foram injetados, em condições idênticas, mais três animais de cada um dos quatro grupos, a serem utilizados como substitutos em caso de morte de animais durante o tratamento crônico ou na preparação aguda. Restaram, do total, três ratos do grupo I, dois ratos do grupo II e um rato do grupo IV, que foram utilizados para registro do potencial de ação do músculo gastroenêmico, obtido por estímulo isolado, utilizando-se amplificador Grass p-15 e osciloscópio Tektronix 564; o registro foi feito por câmera quimográfica Grass.

Os resultados obtidos para um mesmo tipo de informação nos quatro grupos foram submetidos à análise de variância a um critério, adotando-se como limite de significância $\mathrm{p}<0,05$, para o qual $F=2,86$.

\section{RESULTA DOS}

A pesagem dos animais, logo após a anestesia pelo hidrato de cloral, mostrou uma média global dos 40 animais de $354,12 \pm 27,23 \mathrm{~g}$, com um coeficiente de variabilidade de Pearson de $\mathbf{7 , 5} \%$. Os quatro grupos que entraram na experiência apresentaram peso médio semelhante (Tabelas 1 a 4 e Gráfico 1), não havendo diferença significante de peso entre os grupos $(F=0,11)$.

Dos 40 animais utilizados, a maioria $(62,5 \%)$ apresentou limiar minimo de excitabilidade de $0,1 \mathrm{v}$, sendo que $25 \%$ apresentou respostas a pulsos isolados de apenas $0,05 \mathrm{v}$; os restantes $12,5 \%$ responderam a estímulos superiores a $0,1 \mathrm{v}$. $\mathrm{O}$ limiar máximo de excitabilidade oscilou em torno de 0,2 a $0,3 \mathrm{v}$, utilizando-se, a seguir, para a rotina de experiência, estímulos supramaximais de $0,8 \mathrm{v}$ em todos os animais.

As medidas da força da contração normal, resposta a salva de 10 estímulos tetanizantes, tétano mantido por 15 segundos, facilitação pós-tetânica e dose curarizante apresentaram os resultados expressos nas tabelas 1, 2, 3 e 4. Foi a seguir feita a análise de variância de informação semelhante nos quatro grupos, verificando-se que o comportamento foi idêntico no que diz respeito a estímulo isolado (gráfico 2) com $\mathbf{F}=1,89$, o mesmo acontecendo quando tal estímulo era aplicado após o tétano facilitação pós-tetânica (Gráfico 5 ) com $F=1,22$. 


\begin{tabular}{|c|c|c|c|c|c|}
\hline \multirow[t]{2}{*}{ Peso (g) } & \multicolumn{4}{|c|}{ Contração (g) } & \multirow{2}{*}{$\begin{array}{c}\text { Dose } \\
\text { curarizante } \\
\text { DMT } \\
(\mathrm{mcg} / \mathrm{kg})\end{array}$} \\
\hline & $\begin{array}{l}\text { Estímulo } \\
\text { isolado }\end{array}$ & $\begin{array}{l}\text { Salva est. } \\
\text { tetanizantes }\end{array}$ & Tetanização & F.P.T. & \\
\hline 325 & 10,00 & 40,00 & $\mathbf{5 7 , 5 0}$ & $\mathbf{1 5 , 0 0}$ & 4,62 \\
\hline 370 & 20,00 & 70,00 & 102,50 & 32,50 & 6,08 \\
\hline 350 & 17,50 & 25,00 & 85,00 & 25,00 & 2,14 \\
\hline 310 & 15,00 & 25,00 & 87,50 & 17,50 & 2,42 \\
\hline 400 & 22,50 & 50,00 & 72,50 & 25,00 & 3,13 \\
\hline 340 & 5,00 & 25,00 & 80,00 & 17,50 & 4,41 \\
\hline 360 & 8,75 & 30,00 & 52,50 & 15,00 & 2,78 \\
\hline 350 & 30,00 & 60,00 & 67,50 & 25,00 & 4,00 \\
\hline 380 & 30,00 & 75,00 & 92,50 & 37,50 & 3,29 \\
\hline 365 & 20,00 & 65,00 & 92,50 & 25,00 & 3,42 \\
\hline 355 & 17,87 & 46,50 & 79,00 & 23,50 & 3,62 \\
\hline $\mathrm{s}: \quad \pm 26,25$ & $\pm 8,45$ & $\pm 20,00$ & $\pm 16,21$ & $\pm 7,47$ & $\pm 1,17$ \\
\hline C.V.P.: $7,4 \%$ & $47,0 \%$ & $43,0 \%$ & $20,5 \%$ & $\mathbf{3 1 , 5 \%}$ & \\
\hline
\end{tabular}

Tabela 1 - Resultados obtidos em ratos machos, conservados para fins de controle, anestesiados pelo hidrato de cloral $300 \mathrm{mg} / \mathrm{kg} \mathrm{i.p.}$

\begin{tabular}{|c|c|c|c|c|c|}
\hline \multirow[t]{2}{*}{ Peso (g) } & \multicolumn{4}{|c|}{ Contração (g) } & \multirow{2}{*}{$\begin{array}{c}\text { Dose } \\
\text { curarizante } \\
\text { DMT } \\
(\mathrm{mcg} / \mathrm{kg})\end{array}$} \\
\hline & $\begin{array}{c}\text { Estímulo } \\
\text { isolado }\end{array}$ & $\begin{array}{l}\text { Salva est. } \\
\text { tetanizantes }\end{array}$ & Tetanização & F.P.T. & \\
\hline 360 & 18,75 & 50,00 & 70,00 & 18,75 & 2,78 \\
\hline 385 & 22,50 & 65,00 & 70,00 & 22,50 & 1,95 \\
\hline 340 & 17,50 & 50,00 & 75,00 & 22,50 & 3,67 \\
\hline 330 & 27,50 & 65,00 & 85,00 & 32,50 & 3,03 \\
\hline 380 & 20,00 & 65,00 & 90,00 & 32,50 & 3,95 \\
\hline 340 & 15,00 & 40,00 & 65,00 & 20,00 & 3,67 \\
\hline 305 & 27,50 & 75,00 & 112,50 & 35,00 & 4,92 \\
\hline 410 & 17,50 & 50,00 & 70,00 & 27,50 & 3,66 \\
\hline 335 & 20,00 & 45,00 & 67,50 & 25,00 & 2,99 \\
\hline 315 & 17,50 & 47,50 & 62,50 & 20,00 & 6,35 \\
\hline 350 & 20,37 & 55,25 & 76,75 & 25,62 & 3,69 \\
\hline $\mathrm{s}: \quad \pm 33,16$ & $\pm 4,25$ & $\pm 11,33$ & $\pm 15,23$ & $\pm 5,92$ & $\pm 1,21$ \\
\hline C.V.P.: $9,0 \%$ & $20,5 \%$ & $20,5 \%$ & $19,5 \%$ & $23,0 \%$ & $32,5 \%$ \\
\hline
\end{tabular}

Tabela 2 - Resultados obtidos em ratos machos submetidos à administração crônica de soro fisiológico, na dose $1 \mathrm{ml}$ i.p./dia. 


\begin{tabular}{|c|c|c|c|c|c|}
\hline \multirow[t]{2}{*}{ Peso (g) } & \multicolumn{4}{|c|}{ Contração (g) } & \multirow{2}{*}{$\begin{array}{c}\text { Dose } \\
\text { curarizante } \\
\text { DMT } \\
(\mathrm{mcg} / \mathrm{kg})\end{array}$} \\
\hline & $\begin{array}{l}\text { Estímulo } \\
\text { isolado }\end{array}$ & $\begin{array}{l}\text { Salva est. } \\
\text { tetanizantes }\end{array}$ & Tétano & F.P.T. & \\
\hline 355 & 21,25 & 40,00 & 62,50 & 27,50 & 2,82 \\
\hline 350 & 13,75 & 25,00 & 30,00 & 10,00 & 4,29 \\
\hline 375 & 13,75 & 43,75 & 67,50 & 20,00 & 4,67 \\
\hline 365 & 27,50 & 25,00 & 50,00 & 12,50 & 3,90 \\
\hline 385 & 6,25 & 40,00 & 55,00 & 27,50 & 4,79 \\
\hline 370 & 22,50 & 45,00 & 70,00 & 23,75 & 2,70 \\
\hline 350 & 15,00 & 45,00 & 77,50 & 22,50 & 4,29 \\
\hline 385 & 12,50 & 47,50 & 77,50 & 22,50 & 3,90 \\
\hline 345 & 15,00 & 45,00 & 70,00 & 22,50 & 3,62 \\
\hline 290 & 7,50 & 16,25 & 31,25 & 10,00 & 5,17 \\
\hline 357 & 15,50 & 37,25 & 59,12 & 19,87 & 4,01 \\
\hline $\pm 27,60$ & $\pm 6,59$ & $\pm 10,77$ & $\pm 17,38$ & $\pm 6,67$ & $\pm 0,80$ \\
\hline C.V.P.: $7,5 \%$ & $42,5 \%$ & $29,0 \%$ & $29,0 \%$ & $23,5 \%$ & $19,5 \%$ \\
\hline
\end{tabular}

Tabela 3 - Resultados obtidos em ratos machos submetidos à administração crônica do DMT na dose de $2,8 \mathrm{mcg} / \mathrm{kg} /$ dia i.p., anestesiados pelo hidrato de cloral $300 \mathrm{mg} / \mathrm{kg}$ i.p.

\begin{tabular}{|c|c|c|c|c|c|c|}
\hline \multirow{2}{*}{\multicolumn{2}{|c|}{ Peso (g) }} & \multicolumn{4}{|c|}{ Contração (g) } & \multirow{2}{*}{$\begin{array}{c}\text { Dose } \\
\text { curarizante } \\
\text { DMT } \\
(\mathrm{mcg} / \mathrm{kg})\end{array}$} \\
\hline & & $\begin{array}{l}\text { Estímulo } \\
\text { isolado }\end{array}$ & $\begin{array}{l}\text { Salva est. } \\
\text { tetanizantes }\end{array}$ & Tetanização & F.P.T. & \\
\hline & 355 & 10,00 & 37,50 & 58,75 & 20,00 & 4,23 \\
\hline & 310 & 5,00 & 25,00 & 47,50 & 15,00 & 4,03 \\
\hline & 345 & 12,50 & 35,00 & 67,50 & 20,00 & 5,07 \\
\hline & 365 & 12,50 & 33,75 & 62,50 & 25,00 & 3,42 \\
\hline & 335 & 12,50 & 16,25 & 30,00 & 12,50 & 5,97 \\
\hline & 380 & 20,00 & 40,00 & 65,00 & 25,00 & 5,26 \\
\hline & 380 & 12,50 & 42,50 & 67,50 & 25,00 & 6,58 \\
\hline & 340 & 17,50 & 55,00 & 72,50 & 30,00 & 8,09 \\
\hline & 360 & 25,00 & 57,50 & 85,00 & 30,00 & 5,56 \\
\hline & 375 & 12,50 & 27,50 & 60,00 & 20,00 & 6,67 \\
\hline $\bar{x}:$ & 354,50 & 14,00 & 37,00 & 61,62 & 22,25 & 5,48 \\
\hline & $\pm 22,41$ & $\pm 5,55$ & $\pm 12,74$ & $\pm 14,74$ & $\pm 5,82$ & $\pm 1,40$ \\
\hline C.V.P.: & $\therefore \quad 6 \%$ & $39,5 \%$ & $34,0 \%$ & $23,5 \%$ & $26,0 \%$ & $25,5 \%$ \\
\hline
\end{tabular}

Tabela 4 - Resultados obtidos em ratos machos submetidos à administracão crônica do DMT na dose de $14 \mathrm{mcg} / \mathrm{kg} /$ dia i.p., anestesiados pelo hidrato de cloral $300 \mathrm{mg} / \mathrm{kg}$ i.p. 


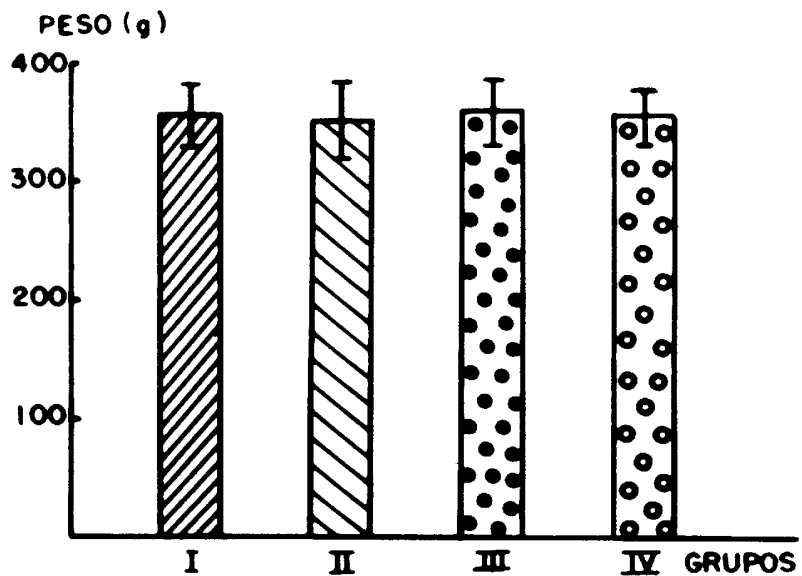

Gráfico 1 - Peso médio dos animais que entraram na experiência.

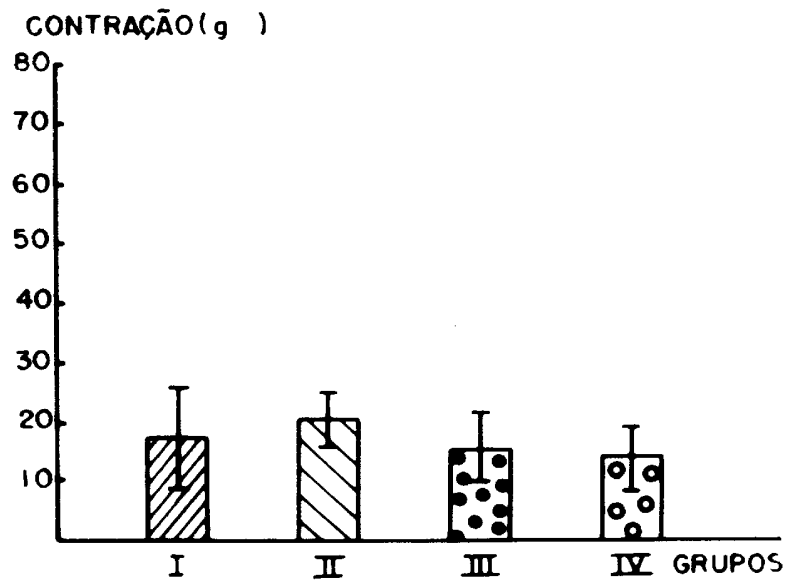

Gráfico 2-Contração média em resposta a estímulos isolados supra-maximais repetidos a intervalos de 3 seg. 


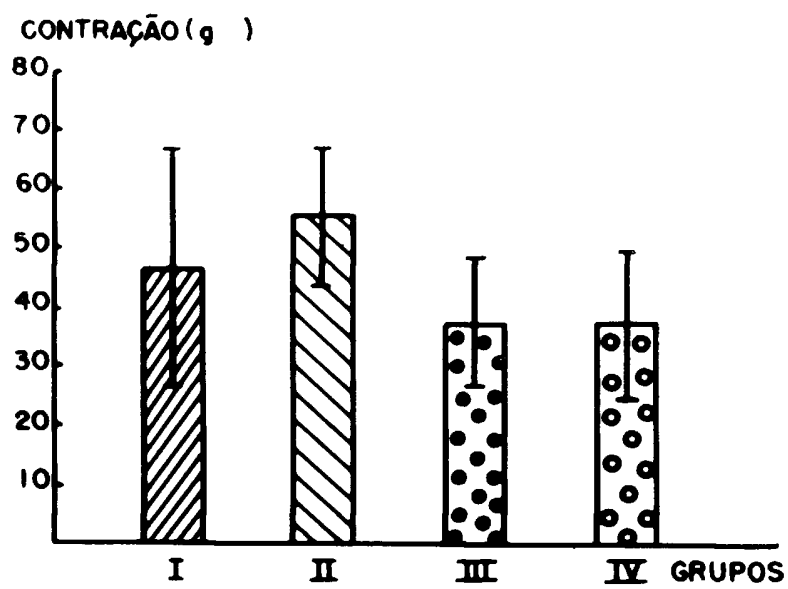

Gráfico 3 - Contracão média em resposta a salvas de 10 estímulos supra-maximais tetanizantes (70 pulsos/seg) repetidos a intervalos de 3 seg.

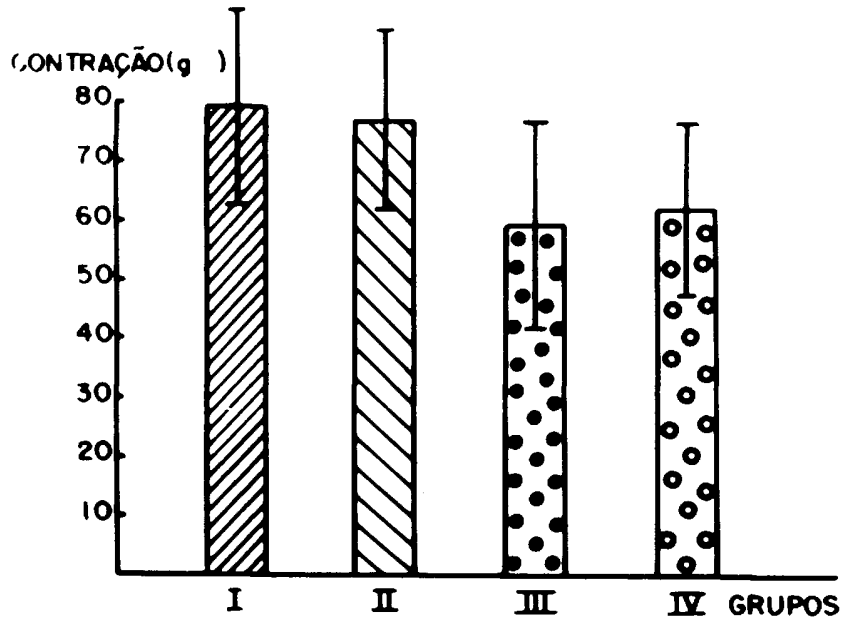

Gráfico 4 - Contração média em resposta a tétano provocado por estimulos supra-maximais (70 pulsos/seg) mantidos por 15 seg. 


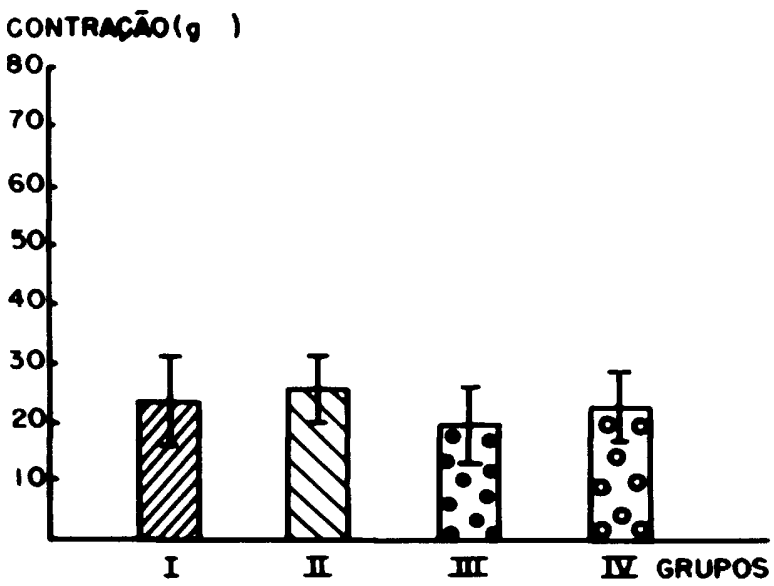

Gráfico 5 - Contração média na facilitacão pós-tetânica em resposta a estimulos isolados supramaximais repetidos a intervalos de 3 seg.

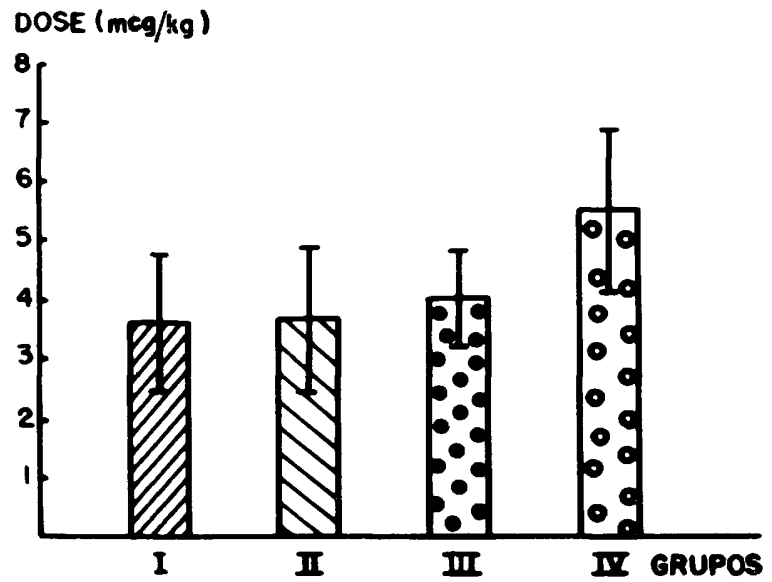

Gráfico 6 - Dose curarizante média do dimetileter da d-tubocurarina em injeção intravenosa. 
Entretanto, os dois grupos tratados com curare apresentaram resposta significativamente diminuída ao estímulo tetanizante. No caso da salva de dez estímulos tetanizante (Gráfico 3) a contração foi aproximadamente $30 \%$ menor do que nos grupos controle $(\mathbf{F}=3,74)$. Quando a tetanização foi mantida (Gráfico 4), a contração média dos grupos III e IV respectivamente - 59,12 g e 61,62 $\mathrm{g}$ - foi aproximadamente $20 \%$ menor do que os grupos I e II, respectivamente $79,00 \mathrm{~g}$ e $76,75 \mathrm{~g}$ $(F=4,10)$.

A avaliação da dose curarizante revelou, para os quatro grupos, os valores médios de: Grupo I - 3,62 $\mathrm{mcg} / \mathrm{kg}$; Grupo II $-3,69 \mathrm{mcg} / \mathrm{kg}$; Grupo III - 4,01 $\mathrm{mcg} / \mathrm{kg}$; Grupo IV - $5,48 \mathrm{mcg} / \mathrm{kg}$ (Gráfico 6). A análise de variância most)ou diferenca significativa entre tais resultados $(F=5,22)$.

Os registros de potencial de ação, bem como a análise do efeito farmacológico da neostigmina não revelaram diferença significativa entre os vários grupos.

\section{COM ENTARIOS}

Os resultados obtidos na presente experiência e os citados em trabalho anterior ${ }^{14}$ sugerem diminuição da força muscular de ratos submetidos a administração crônica do curare. Realmente, em condições de maior solicitação (estímulos tetanizantes) os ratos tratados com curare apresentaram contração significativamente mais fraca de que os do grupo controle (Tabelas 1, 2, 3 e 4 e Gráficos 3 e 4). Tal resposta era esperada, em se considerando as hipóteses de que: $(a)$ ocorreria acúmulo da droga no organismo ou $(b)$ o uso repetido do curare diminuiria a capacidade de transmissão da junção neuromuscular.

Por outro lado, já havia sido sugerido ${ }^{14}$ que em ratos não anestesiados, o bloqueio causado por uma mesma dose de DMT, administrada diariamente, torna-se progressivamente menor. $\mathrm{Na}$ presente experiência, a tolerância ao DMT foi avaliada em animais anestesiados, comparando-se a dose curarizante de um grupo submetido previamente a administração diária da droga em relação a um grupo controle. Os resultados obtidos foram comparáveis aos do experimento anterior, pois a dose curarizante média dos animais tratados com DMT em altas doses foi de $5,48 \pm 1,40 \mathrm{mcg} / \mathrm{kg}$, bem maior do que o grupo controle injetado com soro fisiológico, com $3,69 \pm 1,21 \mathrm{mcg} / \mathrm{kg}$ e o grupo controle não manuseado, com $3,62 \pm 1,17 \mathrm{mcg} / \mathrm{kg}$ (ver Tabelas 1,2 e 4 e Gráfico 6 ).

Assim, embora se tivesse comprovado a hipótese de que a presença freqüente de uma droga curarizante na circulação seja responsável pela diminuição da força muscular no miastênico, a sensibilidade à administração aguda do curare varia de maneira oposta, pois o miastênico é extremamente sensivel à injeção venosa de curare.

Tais achados, aliados ao grande número de descobertas que se tem feito em relação à imunologia e patogenese da miastenia grave $1,5,7,9,11,13$, ressalvada a diferença de espécie, faz supor que a presença de droga curarizante no sangue, sintetizada no timo, pode contribuir um pouco na fraqueza muscular do miastênico, mas é fator secundário e de menor importância. 


\section{R E S U M}

Baseados em teoria discutivel segundo a qual a miastenia grave é provocada pela presença no sangue de substância curarizante liberada pelo timo, tentou-se reproduzir um modelo experimental. Foram utilizados 40 ratos, criados nas mesmas condições, nascidos no mesmo dia, pesando ao redor de $350 \mathrm{~g}$ e divididos em 4 grupos: o Grupo I não foi manuseado; no Grupo II foi injetada solução fisiológica, $1 \mathrm{ml}$ i.p. durante 6 semanas; no Grupo III foi injetada a dimetil tubocurarina (DMT) $2,8 \mathrm{mcg} / \mathrm{kg}$ i.p., durante o mesmo tempo; no Grupo IV $14 \mathrm{mcg} / \mathrm{kg}$ da mesma droga foram injetados i.p. Uma semana após a última injeção i.p. os ratos, anestesiados, foram preparados para registro neuromuscular. O nervo ciático foi estimulado nas freqüências de 0,33 pulsos/seg, 70 pulsos/seg (séries de 10 pulsos a intervalos de $3 \mathrm{seg}$ ), 70 pulsos/seg (mantidos por $15 \mathrm{seg}$ ) e novamente 0,33 pulsos/seg logo após a tetanização. A dose curarizante de DMT foi determinada por uma "terceira parte cega" quando $80 \%$ do bloqueio era alcançado.

Quando apenas 10 estímulos em alta freqüência foram aplicados ao nervo, foi observada diferença significativa $(p<0.05)$ na resposta: Grupo $I, 46,50 \pm$ 20,00 $\mathrm{g}^{+}$; Grupo II, 55,25 $\pm 11,33 \mathrm{~g}^{+}$; Grupo III, 37,25 $\pm 10,77 \mathrm{~g}^{+}$; Grupo IV $37,00 \pm 12,74 \mathrm{~g}^{+}$. Diferenças significantes de força muscular foram também observadas após a tetanização mantida: Grupo I, 79,00 $\pm 16,21 \mathrm{~g}^{+}$; Grupo II, 76,75 \pm 15,23 $\mathrm{g}^{+}$; Grupo III, 59,12 $\pm 17,38 \mathrm{~g}^{+}$; Grupo IV, 61,62 $\pm 14,74 \mathrm{~g}$.

Doses significamente mais altas de curare i.v. foram necessárias no grupo injetado diariamente com a maior dose de curare do que em qualquer dos outros grupos ( $\mathrm{p}<0,01$ ): Grupo I, 3,62 $\pm 1,17 \mathrm{mcg} / \mathrm{kg}$; Grupo II, $3,69 \pm 1,21 \mathrm{mcg} / \mathrm{kg}$; Grupo III, $4,01 \pm 0,80 \mathrm{mcg} / \mathrm{kg} ;$ Grupo IV, $5,48 \pm$ $1,40 \mathrm{mcg} / \mathrm{kg}$.

Tais resultados mostram que a administração crônica do curare leva ao enfraquecimento físico e hiposensibilidade à droga. Isto sugere que embora a existência de substância curarizante no sangue humano possa realmente contribuir para a fraqueza muscular do paciente miastênico, o curare sangüíneo não tem importância fundamental na patogênese da síndrome, pois o miastênico é altamente sensível à injeção de qualquer substância curarizante.

\section{S U M M A R Y}

Tolerance to curarizing drug induced by chronic administration: an experimental study

The trial is a temptative experimental model of myasthenia gravis based on a doubtful theory whereby this disease is atributed to circulating curare in blood. Forty rats kept under the same conditions, born on the same day and weighing around $350 \mathrm{~g}$ entered the trial, divided in four groups. Group I was not handled, Group II was injected with saline, $1 \mathrm{ml}$ i.p. Group III was 
submitted to dimethyl tubocurarine iodide (DMT) $2,8 \mathrm{mcg} / \mathrm{kg}$ i.p. and Group IV received $14 \mathrm{mcg} / \mathrm{kg}$, all solutions being administered daily for a six-week period. One week after the last rats were anesthetized and prepared for cyatic-gastrocnemius neuro-muscular recording. Monopolar pulses were given at frequencies of $0.33 \mathrm{p} / \mathrm{sec}, 70 \mathrm{p} / \mathrm{sec}$, (trains of 10 pulses at 3 sec intervals), $70 \mathrm{p} / \mathrm{sec}$ (kept for $15 \mathrm{sec}$ ) and again $0.33 \mathrm{p} / \mathrm{sec}$ right after tetanus. Curarizing effective dose of DMT was determined by a "third part blind" when a $80 \%$ block was attained.

When only 10 high frequency stimuli were applied to the nerve, a significant difference $(p<0.05)$ in response was observed: Group $I, 46.50 \pm$ $20.00 \mathrm{~g}^{+}$; Group II, $55.25 \pm 11.33 \mathrm{~g}^{+}$; Group III, $37.25 \pm 10.77 \mathrm{~g}^{+}$; Group IV, $37.00 \pm 12.74 \mathrm{~g}^{+}$. Significant differences in muscular force were also observed with sustained tetanus: Group I, $79.00 \pm 16.21 \mathrm{~g}^{+}$; Group II, $76.75 \pm 15.23 \mathrm{~g}^{+}$; Group III, $59.12 \pm 17.38 \mathrm{~g}^{+}$; Group IV, $61.62 \pm$ $14.74 \mathrm{~g}$.

Significant higher doses of curare i.v. were necessary in the group injected daily with the highest dose of curare than in any other group $(\mathrm{p}<$ 0.01): Group I, $3.62 \pm 1.17 \mathrm{mcg} / \mathrm{kg}$; Group II, $3.69 \pm 1.21 \mathrm{mcg} / \mathrm{kg}$; Group III, $4.01 \pm 0.80 \mathrm{mcg} / \mathrm{kg}$; Group IV, $5.48 \pm 1.40$.

These results show that chronic administration of curare leads to physical weakness and hyposensitivity to the drug, thus suggesting that although the existence of a curarizing drug in the human blood may in fact contribute for the muscular weakness of the myasthenic patient, the blood curare does not play a major role in the pathogenesis of the syndrome since the myasthenic patient is highly sensitive to the injection of any curare.

\section{R E F E R E N I A S}

1. ASSIS, J. L. - Afecções musculares agudas e subagudas. Rev. Paulista de Medicina 75:63, 1969.

2. CANNON, M. B. \& ROSENBlueth, N. - The Supersensitivity of Denervated Structures. MacMillan Co., New York, 1949.

3. CHURCHILL-DAVIDSON, H. C. - Myasthenic crisis. Therapeutic use of d-tubocurarine. Lancet 273:1221, 1957.

4. DESMEDT, J. E. - Presynaptic mechanisms in myasthenia gravis. Ann. N. Y. Acad. Sci. 135:209, 1966.

5. EDITORIAL - Pathogenesis of myasthenia gravis. Br. Med. J. 2:1, 1971.

6. EMMELIN, R. - Supersensitivity following pharmacological denervation. Pharmacological Rev. 13:17, 1961.

7. GOLDSTEW, G. - The thymus and neuromuscular functions. A substance in thymus which causes myositis and myasthenic neuromuscular block in guinea pigs. Lancet 2:119, 1968.

8. GROB, P. - Neuromuscular pharmacology. Ann. Rev. Pharmacol. 1:239, 1961.

9. KALDEN, J. R. \& IRVINE, W. S. - Experimental myasthenia gravis. Lancet $2: 638,1969$.

10. NASTUK, W. L. \& PLESCIN, O. J. - Current status of research on myasthenia gravis. Ann. N. Y. Acad. Sci. 135:664, 1966.

11. SARAIVA, S. - Aspectos auto-imunes da miastenia grave. Rev. Hosp. Clin. Fac. Med. São Paulo 25:181, 1970. 
12. SHARPLESS, S. K. - Reorganization of function in the nervous system: use and disuse. Ann. Rev. Physiol. 26:357, 1964.

13. VETTERS, J. M. \& SIMPSON, J. A. - Experimental myasthenia gravis. Lancet $2: 28,1969$.

14. ZANINI, A. C. - Alterações funcionais da junção neuromuscular provocadas em ratos pela administração díria e prolongada de um agente curarizante. Arq. Neuro-Psiquiat. (São Paulo) 25:112, 1967.

Departamento de Terapêtica Clinica - Faculdade de Medicina - Caixa Postat 2921 - 01000 São Paulo, SP - Brasil. 